\title{
Inequidades en el diagnóstico de anomalías congénitas mayores en recién nacidos en Cali, Colombia.
}

\author{
Fabián Andrés Ruiz-Murcia 1,2,3, Andrés Fandiño-Losada1,2,4,Julián Ramirez-Cheyne 1,2,5, \\ Carolina Isaza 1,2,5,6, Wilmar Saldarriaga. 1,2,5,6,7,8 \\ ${ }^{1}$ Universidad del Valle, Cali, Colombia. ${ }^{2}$ Grupo de Investigación en Malformaciones Congénitas Perinatales y Dismorfo- \\ logía de la Universidad del Valle, MACOS. ${ }^{3}$ Grupo Estudiantil de Genética Clínica, Universidad del Valle, Cali, Colombia. \\ ${ }^{4}$ Escuela de Salud Pública, Facultad de Salud, Universidad del Valle, Cali, Colombia. ${ }^{5}$ Departamento de Morfología, \\ Escuela de Ciencias Básicas, Facultad de Salud, Universidad del Valle, Cali Colombia. ${ }^{6}$ Laboratorio de Genética Médica \\ "Carolina Isaza, Clínica DIME, Cali, Colombia. ${ }^{7}$ Departamento de Ginecología y Obstetricia, Escuela de Medicina, Uni- \\ versidad del Valle, Cali, Colombia. ${ }^{8}$ Gineco-Obstetra, Hospital Universitario del Valle "Evaristo García"
}

\section{RESUMEN}

Antecedentes: Las anomalías congénitas mayores (ACM) están presentes en el 2-3\% de los nacidos vivos. La mayoría de ellas diagnosticables mediante ecografía prenatal (EcoPN). Los países en desarrollo presentan inequidades en el acceso al procedimiento. Objetivos: Determinar las inequidades existentes en la realización de EcoPN en las madres de recién nacidos (RN) con ACM hospitalizados en dos unidades de cuidado intensivo neonatal (UCIN) e identificar las inequidades en el diagnóstico de ACM en las madres a quienes les fue realizada al menos una EcoPN. Métodos: Estudio transversal en RN con ACM diagnosticables por EcoPN, hospitalizados en dos UCIN de Cali, Colombia, entre 2005 y 2009. Se determinó el índice de concordancia (kappa) entre variables sociodemográficas y el diagnóstico de ACM prenatal y definitivo. Se determinaron las asociaciones entre ausencia de EcoPN y del diagnóstico de ACM con factores sociodemográficos y aseguramiento en salud. Resultados: Se incluyeron 404 casos que presentaron 573 anomalías congénitas. El 51,7\% (IC95\% 46,7\%-56,7\%) tuvieron al menos una EcoPN; de éstos el $31,1 \%$ (IC95\% 24,9\%-37,9\%) no tuvo diagnóstico de ACM. La ausencia de EcoPN estuvo asociada a la afiliación al régimen de salud subsidiado, la no afiliación al sistema de salud, a las madres procedentes de municipios diferentes a la capital de la provincia, de otras provincias y del área rural. Hubo asociaciones similares para la ausencia de diagnóstico de alguna ACM en la EcoPN. Conclusiones: Se detectaron inequidades en la realización EcoPN y en el diagnóstico de ACM en las madres de mayor vulnerabilidad social y demográfica. Se debe mejorar el acceso a los servicios de EcoPN en Colombia para cerrar estas disparidades sociales.

\section{PALABRAS CLAVE: Anomalías congénitas, diagnóstico prenatal, ultrasonografía prenatal, inequidades sociales, inequidades geográficas, sistema de salud}

\section{SUMMARY}

Background: Major Congenital Anomalies (MCA) are present in 2-3\% of live births. Most of these are diagnosable by prenatal ultrasound (PNUS). Developing countries have inequities in access to this screening test. Objectives: To determine existing inequities access to PNUS in mothers of newborns (NB) with MCA hospitalized in two neonatal intensive care units (NICU) and to identify inequities in the diagnosis of MCA among mothers to whom it was made at least one PNUS. Methods: Cross-sectional study in NB with MCA diagnosable by PNUS hospitalized in two NICUs of Cali, Colombia, between 2005 and 2009. The index of agreement (kappa) between prenatal diagnosis and definitive MCA was calculated. It was 
established associations between absence of PNUS and diagnosis of MCA with socio-demographic factors and health insurance schemes. Results: 404 cases with 573 MCA were included. $51.7 \%$ (95\% Cl: 46.7\%$56.7 \%)$ had at least one PNUS; of these $31.1 \%$ (95\% Cl: $24.9 \%-37.9 \%)$ had no diagnosis of MCA. The absence of PNUS was associated with affiliation to the subsidized health scheme, no-affiliation to the health system, mothers from municipalities different to the capital of the province, from other provinces and from rural areas. There were similar associations for the absence of a diagnosis of MCA in PNUS. Conclusions: It was detected inequities in access to the PNUS and in diagnosis of MCA for mothers of greater social and demographic vulnerability. The access to services of PNUS in Colombia should been improved, in order to reduce these social disparities.

\section{KEY WORDS: Congenital anomalies, prenatal diagnosis, prenatal ultrasonography, social inequi- ties, geographic inequalities, health system}

\section{INTRODUCCIÓN}

Las anomalías congénitas mayores (ACM) están presentes en el $2-3 \%$ de los nacidos vivos (1-4), estando entre las principales causas de la mortalidad neonatal $(2,3)$. La ecografía prenatal de rutina (EcoPN) ha sido estandarizada para detectar anomalías congénitas, embarazos múltiples, desórdenes del crecimiento fetal, alteraciones en la placenta, así como también para establecer la edad gestacional $(3,5)$. La mayoría de ACM pueden ser diagnosticadas antes del nacimiento por medio de la EcoPN, lo cual mejora el pronóstico de los recién nacidos (RN) con anomalías congénitas, dado que esto permite la realización de intervenciones en útero, la programación de la terminación del embarazo, la preparación del equipo neonatal para la atención del RN y la preparación de la familia para tener un nuevo miembro con condiciones especiales $(2,4,5)$.

El diagnóstico prenatal de anomalías congénitas debe aproximarse al $100 \%$ de los casos. En centros de referencia, con los equipos adecuados y especialistas en perinatología y/o medicina materno-fetal, la exactitud diagnóstica debe ser cercana a la ideal (5). Sin embargo, en estudios en población general de bajo riesgo con técnicas ecográficas prenatales básicas, la sensibilidad disminuye.

En Europa el porcentaje global de detección de las ACM es del $55 \%$, variando según el tipo de defecto congénito, desde una sensibilidad del $88,3 \%$ para los casos de defectos del sistema nervioso central hasta una sensibilidad del $38,8 \%$ para los casos de cardiopatías congénitas (6). En un estudio efectuado en América del Sur, se encontró una sensibilidad global del tamizaje con EcoPN del $56 \%$, variando desde el $80 \%$ de casos detectados para los casos de espina bífida, malformaciones renales y de las vías urinarias, anencefalia, defectos de pared abdominal e hidrocefalia, hasta solamente el $25 \%$ de los casos detectados de labio fisurado y anomalías de las extremidades (7). Por otro lado, en estudios en Colombia la efectividad de la EcoPN para diagnosticar las ACM está lejos de la ideal.
Por ejemplo, la detección ecográfica de $\mathrm{ACM}$ en tres hospitales de Bogotá fue solamente del 32,5\% (8) y en dos instituciones de salud de Cali, la sensibilidad de la EcoPN para detectar al menos una ACM fue del $79,2 \%$ (9).

En estudios en países en desarrollo, se ha establecido que las inequidades en el acceso a la EcoPN y en el diagnóstico de ACM de las ecografías obstétricas están relacionadas con el nivel socioeconómico, el tipo de aseguramiento en salud y la etnia; encontrándose que las personas pertenecientes a los estratos socioeconómicos más altos y a servicios de salud privados tienen mayores probabilidades de un diagnóstico de ACM en la EcoPN (10). Por otro lado, las mujeres de bajos recursos económicos, bajos niveles educativos, pertenecientes a las minorías étnicas o que viven en áreas rurales tienen menos probabilidades de acceder a los servicios de tamizaje prenatal (11-13).

En los países en desarrollo, los servicios de atención prenatal, entre éstos la EcoPN, no tienen suficiente cobertura poblacional y se presentan inequidades socio-económicas y regionales respecto a la cobertura de los servicios prenatales, afectando a las personas más pobres y en las áreas rurales $(14,15)$. Sin embargo, los estudios sobre inequidades en la utilización de los servicios de atención prenatal en países en desarrollo no se han enfocado en la utilización de la ecografía obstétrica, con excepción de un estudio brasilero que mostró que no existían diferencias socioeconómicas o raciales en la realización de al menos una EcoPN durante el embarazo, aunque sí existían diferencias en el número de EcoPN realizadas (16).

Se debe tener en cuenta que en América Latina existe una gran variedad de sistemas de salud, con una participación diferente del Estado y de sectores privados en cada país (17). Por lo tanto, es necesario explorar las inequidades en el acceso a EcoPN en el contexto colombiano. Los objetivos de este estudio fueron: A. Determinar las inequidades existentes en la realización de EcoPN en las madres de recién nacidos (RN) con $A C M$, hospitalizados en 
dos unidades de cuidado intensivo neonatal (UCIN) de Cali, Colombia, entre 2005-2009, y B. Identificar las inequidades existentes en el diagnóstico de ACM en las madres a quienes se les realizó al menos una EcoPN durante su embarazo.

\section{PACIENTES Y MÉTODOS}

Se efectuó un estudio transversal en recién nacidos (RN) hospitalizados en la UCIN de dos instituciones de tercer nivel de Cali, Colombia. La primera institución fue el Hospital Universitario del
Valle (HUV), centro de referencia para la red pública del suroccidente colombiano, y la segunda fue la Fundación Valle del Lili (FVL), una entidad privada que principalmente atiende pacientes con afiliación al régimen contributivo y pacientes remitidos de otras regiones del país. El estudio incluyó todos los pacientes hospitalizados en las UCIN de ambas instituciones desde enero de 2005 hasta diciembre del 2009 (5 años), en quienes se había confirmado el diagnóstico de alguna ACM diagnosticable con EcoPN (Tabla I).

\section{FRECUENCIAS ABSOLUTASY RELATIVAS DE LOS DIAGNÓSTICOS DE ANOMALÍAS CONGÉNITAS MAYORES INCLUIDAS EN EL ESTUDIO}

\begin{tabular}{lr}
\hline Tipos de defecto y diagnósticos & Núm \\
\hline Cardiopatías: $\mathbf{2 1 5}$ & 47 \\
Comunicación interventricular & 24 \\
Transposición de grandes vasos & 14 \\
Atresia pulmonar & 12 \\
Tetralogía de Fallot & 118 \\
Cardiopatía compleja y otras*
\end{tabular}

Anomalía gastrointestinal: 39

Atresia esofágica

Anomalías del tubo neural: 34

Otros

Anomalías renales: 32

Cromosomopatías: $\mathbf{3 1}$

Anomalías de las extremidades: $\mathbf{2 6}$

Anomalías de la pared abdominal: 26

Otras anomalías: 147

$\begin{array}{ll}\text { Síndrome dismórfico } & 20\end{array}$

$\begin{array}{ll}\text { Hernia Diafragmática } & 17\end{array}$

Otros

*Todos los casos con comunicación interatrial están incluidos en las cardiopatías complejas. 
En los casos en los cuales la madre fue sometida a EcoPN, el diagnóstico prenatal del RN fue valorado frente al diagnóstico final en la UCIN y se definieron las siguientes categorías con respecto a la exactitud diagnóstica de la EcoPN: 1) Sin diagnóstico, en los recién nacidos con ACM diagnosticables mediante EcoPN para quienes no se hizo un diagnóstico prenatal aun habiéndose realizado al menos una EcoPN; 2) Diagnóstico incompleto o inexacto, para los casos en los cuales la EcoPN detectó una o varias anomalías, pero que además el RN presentaba también defectos adicionales no detectados durante la EcoPN que pudieron haberse detectado, por ejemplo la comunicación interventricular o la tetralogía de Fallot; 3) Diagnóstico completo y exacto, en los casos para quienes todos los hallazgos de la EcoPN fueron encontrados en los RN y que no tenían otros defectos diagnosticables con la EcoPN.

Se obtuvo la información de los RN mediante la revisión de las historias clínicas de los pacientes. Se desarrolló un formato de recolección de datos en la base de datos de Epi-Info ${ }^{\mathrm{TM}}$ 3.5.3, que incluyó el diagnóstico prenatal y el diagnóstico definitivo de las ACM de los RN en la UCIN, junto con los datos socio-demográficos de la madre. Se determinó el índice de concordancia (k, kappa) (18) entre el número de diagnósticos realizados mediante la EcoPN y el número de diagnósticos postnatales realizados en la UCIN. Además, se calculó la proporción de ocurrencia de los eventos resultado (No EcoPN y No Diagnóstico en la EcoPN) con sus intervalos de confianza del 95\% (IC95\%). Finalmente, adoptando un análisis del tipo casos y controles, se realizaron regresiones logísticas múltiples para explicar las asociaciones entre la ausencia de EcoPN y la ausencia del diagnóstico en la EcoPN (en los pacientes que sí tuvieron EcoPN) como variables resultado, con las variables socio-demográficas (edad de la madre, tipo de seguro de salud, municipio de residencia y área de residencia), expresadas como la oportunidad relativa (OR) del resultado.

Los comités de ética de la Universidad del Valle y de ambas instituciones de salud (HUV y FVL) aprobaron esta investigación.

\section{RESULTADOS}

Durante el periodo estudiado, en las UCIN de las dos instituciones se encontraron 416 pacientes hospitalizados con al menos un defecto congénito diagnosticable por ecografía prenatal, de los cuales $404(97,1 \%)$ tenían información sobre la realización, o ausencia de ecografías obstétricas y los diagnósticos reportados por éstas.
Los 404 pacientes incluidos en este estudio presentaron 573 diagnósticos en UCIN de ACM detectables por ecografía obstétrica, para un promedio de 1,64 (IC95\% 1,52-1,76) diagnósticos por paciente. La mayoría de los diagnósticos correspondieron a cardiopatías congénitas, afectando al 53,2\% de los pacientes. Las otras anomalías reportadas en orden decreciente fueron: defectos gastrointestinales, defectos del tubo neural, defectos renales, cromosomopatías, defectos en extremidades y queilopalatosquisis, afectando del $9,7 \%$ al $5,7 \%$ de los pacientes (Tabla I).

De los pacientes con datos disponibles, el $51,7 \%$ (IC95\% 46,7\%-56,7\%) tuvieron al menos una EcoPN. De los que tuvieron al menos una EcoPN, para el 31,1\% (IC95\% 24,9\%-37,9\%) no se realizó el diagnóstico de ninguna $\mathrm{ACM}$, para el 22,5\% (IC95\% 17,0\%-28,8\%) este diagnóstico fue incompleto o inexacto y para el $46,4 \%$ (IC95\% $39,5 \%-53,4 \%$ ) el diagnóstico prenatal de sus ACM fue exacto. Adicionalmente, se determinó la concordancia entre el número de diagnósticos de ACM por EcoPN y el número de diagnósticos definitivos de ACM en la UCIN mediante el índice kappa, entre los pacientes que tuvieron al menos una EcoPN. De esta forma, el índice kappa de los diagnósticos para todos los pacientes con EcoPN fue regular: $k=0,305$ (IC95\% 0,222-0,371). No hubo diferencias significativas en el índice kappa de los pacientes del régimen de salud contributivo ( $k=0,268$ regular; IC95\% 0,168-0,385) versus el régimen de salud subsidiado ( $\mathrm{k}=0,343$ regular; IC95\% 0,216-0,452). La muestra de la población pobre por atender con EcoPN fue muy pequeña $(n=33)$, entonces el intervalo de confianza del índice kappa no fue informativo.

Mediante análisis de regresión logística, se establecieron los factores de riesgo para la ausencia de EcoPN encontrándose asociaciones significativas entre la falta de la misma y las madres afiliadas al régimen de salud subsidiado o sin afiliación al sistema de salud, las madres procedentes de municipios diferentes a Cali (capital de la provincia del Valle del Cauca) o de municipios de otras provincias y las madres procedentes del área rural (Tabla II). Mientras que para los casos que sí tuvieron EcoPN, se encontró que la ausencia de diagnóstico prenatal está asociada a madres en edades entre 25 a 34 años y entre 35 a 47 años en comparación con las madres de 18 a 24 años; a madres afiliadas al régimen de salud subsidiado y madres sin afiliación al sistema de salud, y a madres procedentes de municipios del Valle del Cauca diferentes a la capital (Cali) y provenientes del área rural (Tabla III). 
Tabla II

DESCRIPCIÓN DE LAS VARIABLES DE ESTUDIOY SUS ASOCIACIONES CRUDAS CON LA AUSENCIA FALTA DE ECOGRAFÍA PRENATAL

\begin{tabular}{|c|c|c|c|c|c|c|}
\hline \multirow[b]{2}{*}{ Variables del estudio } & \multicolumn{2}{|c|}{ Pacientes con ecografía } & \multicolumn{2}{|c|}{ Pacientes sin ecografía } & \multirow[b]{2}{*}{ OR } & \multirow[b]{2}{*}{ (IC95\%) } \\
\hline & $\mathrm{n}$ & $\%$ & $\mathrm{n}$ & $\%$ & & \\
\hline Edad de la madre: & 181 & & 164 & & & \\
\hline 18 a 24 años & 82 & 45,3 & 62 & 37,8 & 1 & \\
\hline 14 a 17 años & 22 & 12,2 & 16 & 9,8 & 0,96 & $(0,47-1,98)$ \\
\hline 25 a 34 años & 55 & 30,3 & 59 & 36,0 & 1,4 & $(0,87-2,32)$ \\
\hline 35 a 47 años & 22 & 12,2 & 27 & 16,4 & 1,62 & $(0,85-3,12)$ \\
\hline Tipo de Seguro de Salud: & 205 & & 183 & & & \\
\hline Contributivo & 82 & 40,0 & 33 & 18,0 & 1 & \\
\hline Subsidiado & 110 & 53,7 & 136 & 74,3 & $3,07^{\star \star \star}$ & $(1,91-4,94)$ \\
\hline Sin Seguro & 13 & 6,3 & 14 & 7,7 & $2,68^{*}$ & $(1,14-6,30)$ \\
\hline Municipio de Residencia: & 182 & & 165 & & & \\
\hline Cali, capital provincial & 87 & 47,8 & 51 & 30,9 & 1 & \\
\hline Otros del Valle del Cauca & 58 & 31,9 & 61 & 37,0 & $1,79^{\star}$ & $(1,09-2,95)$ \\
\hline Fuera del Valle del Cauca & 37 & 20,3 & 63 & 32,1 & $2,44^{\star \star}$ & $(1,42-4,21)$ \\
\hline Área de Residencia: & 205 & & 181 & & & \\
\hline Urbana & 188 & 91,7 & 134 & 74,0 & 1 & \\
\hline Rural & 17 & 8,3 & 47 & 26,0 & $3,9^{\star \star \star}$ & $(2,13-7,05)$ \\
\hline
\end{tabular}

${ }^{*} \mathrm{p}<0,05 ;{ }^{* *} \mathrm{p}<0,01 ;{ }^{* * *} \mathrm{p}<0,001$

Tabla III

MODELOS DE REGRESIÓN LOGÍSTICA AJUSTADOS PARA LA AUSENCIA DE ECOGRAFÍA PRENATAL Y LA AUSENCIA DE DIAGNÓSTICO PRENATAL

\begin{tabular}{|c|c|c|c|c|}
\hline \multirow[b]{2}{*}{ Variables: } & \multicolumn{2}{|c|}{$\begin{array}{l}\text { Modelo 1: } \\
\text { Ausencia de ecografía }\end{array}$} & \multicolumn{2}{|c|}{$\begin{array}{c}\text { Modelo 2: } \\
\text { Ausencia de diagnóstico prenata }\end{array}$} \\
\hline & OR & (IC95\%) & OR & (IC95\%) \\
\hline \multicolumn{5}{|l|}{ Edad de la madre: } \\
\hline 18 a 24 años & 1 & & 1 & \\
\hline 14 a 17 años & 0,68 & $(0,31-1,51)$ & 0,77 & $(0,22-2,76)$ \\
\hline 25 a 34 años & $2,04^{\star}$ & $(1,15-3,62)$ & $2,98^{\star}$ & $(1,17-7,60)$ \\
\hline 35 a 47 años & $2,37^{\star}$ & $(1,08-5,20)$ & $5,07^{\star \star}$ & $(1,47-17,56)$ \\
\hline \multicolumn{5}{|l|}{ Tipo de Seguro de Salud: } \\
\hline Contributivo & 1 & & 1 & \\
\hline Subsidiado & $3,85^{\star \star \star}$ & $(2,16-6,86)$ & $2,63^{*}$ & $(1,09-6,36)$ \\
\hline Sin Seguro & $3,41^{*}$ & $(1,29-8,94)$ & $10,30^{\star \star \star}$ & $(2,49-42,65)$ \\
\hline \multicolumn{5}{|l|}{ Municipio de residencia: } \\
\hline Cali, capital provincial & 1 & & 1 & \\
\hline Otros en Valle del Cauca & 1,32 & $(0,76-2,32)$ & $3,12^{\star \star}$ & $(1,33-7,29)$ \\
\hline Fuera del Valle del Cauca & 1,79 & $(0,94-3,41)$ & 0,98 & $(0,32-3,00)$ \\
\hline \multicolumn{5}{|l|}{ Área de residencia: } \\
\hline Urbana & 1 & & 1 & \\
\hline Rural & $2,83^{\star \star}$ & $(1,37-5,89)$ & 0,52 & $(0,13-2,14)$ \\
\hline
\end{tabular}

Niveles de significancia: ${ }^{*} p<0,05 ;{ }^{* *} p<0,01 ;{ }^{* \star *} p<0,001$ 


\section{DISCUSIÓN}

El diagnóstico prenatal ha evolucionado en paralelo con los avances en las técnicas de ultrasonido, desde 1958, cuando Donald hizo la primera contribución al ultrasonido en humanos, pasando por ecografías en dos y tres dimensiones y el ultrasonido multiplanar y de planos ortogonales, hasta llegar finalmente al ultrasonido con Doppler pulsado. Estos cambios han permitido una aproximación no invasiva a detalles anatómicos mínimos del embrión y el feto, de modo que prácticamente el 100\% de las anomalías congénitas podrían ser diagnosticadas antes del nacimiento (4).

Tras la detección de un defecto congénito en el feto, se realiza un protocolo médico para encontrar la causa, por ejemplo, la toma de una muestra de vellosidad coriónica, líquido amniótico o sangre de cordón umbilical para cariotipo, en casos en los que se sospecha o se quiere descartar alguna anomalía cromosómica $(2,6)$. También hay protocolos médicos para establecer el pronóstico del embarazo, como en los casos de anencefalia o agenesia renal bilateral, y por lo tanto justificar el derecho constitucional existente en algunos países, como Colombia, para interrumpir voluntariamente el embarazo (4); realizar una intervención fetal en útero en casos seleccionados, como la liberación de bandas amnióticas o utilización de láser fetoscópico $(5,19,20)$; definir la mejor vía de finalización del embarazo (20-22) e integrar el grupo de neonatólogos, cirujanos pediátricos y otros especialistas, en casos de manejo complejos como las gastrosquisis $(19,22)$. La realización de determinadas intervenciones que son necesarias si se cuenta con un diagnóstico prenatal exacto, pueden llevar a la disminución de la morbilidad y la mortalidad perinatal, además de preparar a la familia para recibir un miembro con condiciones especiales de vida, quien puede requerir estancias hospitalarias prolongadas y diferentes terapias de apoyo en el período neonatal $(3,4,8)$.

En Colombia, la Resolución 412 del año 2000 emitida por el Ministerio de Salud, era la guía para el proceso de diagnóstico prenatal de ACM, y establecía como norma el tamizaje ecográfico prenatal entre las semanas 20 y 24 para toda mujer embarazada, y la ecografía de detalle anatómico o nivel III para mujeres embarazadas con factores de riesgo o en quienes los hallazgos de la ecografía básica sugieren un defecto congénito. Ésta era la norma que regía en Colombia para el momento del estudio (23). En el 2013, el Ministerio de Salud y Protección Social emite la guía de atención integral a la embarazada en la que se incluye otra ecografía entre las semanas 10 y 6 días y 13 y 6 días (24).

Sin embargo, a pesar del carácter obligatorio de la norma para todos los regímenes de seguridad social, este estudio determinó que el 48,3\% de las madres de RN con ACM diagnosticables por EcoPN y cuyos hijos se encontraban hospitalizados en UCIN, no tuvieron ninguna ecografía en el embarazo, lo cual revela que en Colombia se está lejos de alcanzar la cobertura total en el tamizaje ecográfico prenatal de ACM. También se encontraron inequidades enla realización de las ecografías obstétricas, siendo menor para las madres afiliadas al régimen de salud subsidiado o sin afiliación, para las madres procedentes de municipios diferentes a la capital de la provincia (departamento, en Colombia) o de otras provincias y madres provenientes de áreas rurales, evidenciando que la accesibilidad al control prenatal y a la EcoPN es deficiente para las mujeres pobres y para aquellas que habitan en las áreas más apartadas de los centros urbanos donde se concentran las tecnologías en salud. Del número total de pacientes procedentes de la capital de la provincia (Cali, 138 casos), el $36 \%$ no tuvo EcoPN, y de aquellas madres, el $72 \%$ pertenecían al régimen de salud subsidiado o no estaban afiliadas al sistema de salud, con lo cual este estudio demostró que la población pobre no asegurada tiene acceso limitado a la EcoPN tanto en ciudades capitales como en áreas rurales, siendo el problema más grave en estas últimas zonas. Aunque este tipo de inequidades en el acceso a los servicios de atención prenatalse presenta en países desarrollados, la situación es más grave en los países en desarroIlo, afectando las cifras de mortalidad perinatal en estos países, a expensas de los grupos de población más desfavorecidos al interior de los mismos (10-16).

Además, el análisis de los resultados observados en pacientes que tuvieron EcoPN demostró que hubo un diagnóstico exacto de sus $A C M$ en solo un $46,4 \%$ de los casos, un porcentaje muy bajo considerando que el $100 \%$ de las ACM son diagnosticables mediante ecografía obstétrica.

En este estudio se encontró que las madres entre 25 a 34 años y entre 35 a 47 años, las madres afiliadas al régimen subsidiado o no afiliadas y las madres procedentes de municipios del Valle del Cauca, diferentes a Cali, presentaron un riesgo más alto de carecer de algún diagnóstico prenatal de las ACM de sus hijos, a pesar de haberse realizado al menos una EcoPN (Tabla III). Sin embargo, al tener en cuenta el número de diagnósticos de $\mathrm{ACM}$ en la EcoPN frente a los diagnósticos de ACM en las UCIN no se encontraron diferencias significativas en la concordancia de los diagnósticos entre las madres afiliadas a diferentes regímenes de seguridad social en salud.

De esta forma, se plantea que existen inequidades en la efectividad de la EcoPN para detectar al menos una ACM según el tipo de régimen de salud y el municipio de procedencia de las madres, pero en los casos que se detecta al menos una ACM por la EcoPN, no existen diferencias en la calidad del 
diagnóstico según el régimen de seguridad social en salud.

Lo anterior podría indicar que algunas de las madres de menores recursos socio-económicos o de áreas más apartadas acceden a servicios de ecografía de mala calidad que no permiten diagnosticar las ACM de sus fetos. Desafortunadamente, este estudio no contó con información para probar esta última hipótesis. Adicionalmente, es notable destacar que la mayor probabilidad de ausencia de diagnósticos en la EcoPN para las madres entre 25 a 34 años y entre 35 a 47 años es un hallazgo en contravía con las recomendaciones de las guías nacionales para el control prenatal $(23,24)$, considerando que esos grupos de edad están a mayor riesgo de presentar un producto del embarazo con una ACM; este hecho también podría indicar que existen fallas en la calidad de las ecografías que se realizaron a estas gestantes $(3,7,8)$.

En Colombia, especialmente en las grandes ciudades capitales, un número cada vez mayor de centros de EcoPN básica y avanzada ha incrementado la cobertura de mujeres embarazadas de todos los esquemas de seguridad social, probablemente favoreciendo, en mayor medida, a las mujeres embarazadas afiliadas al régimen de salud contributivo. Sin embargo, en este trabajo se encontró que en todos los grupos sociodemográficos estudiados la cobertura del tamizaje con EcoPN fue menor a la esperada. Por ejemplo, el $31,1 \%$ de las madres del régimen contributivo no accedió a ninguna EcoPN durante su embarazo. Adicionalmente, se evidenció, una cobertura aún menor en madres del régimen de salud subsidiado y en las no aseguradas, encontrándose que dichos grupos de mujeres tenían probabilidades más bajas de realizarse una EcoPN. También, estas mujeres tuvieron una probabilidad mayor de no recibir el diagnóstico de alguna ACM de sus fetos, aun habiéndose realizado al menos una EcoPN, evidenciando disparidades en la calidad de la EcoPN según el régimen de salud o el área de procedencia. Desafortunadamente, en este estudio no se contó con datos sobre el número de ecografías realizadas o la edad gestacional de la realización de las mismas, factores relacionados con la sensibilidad de las ecografías obstétricas $(7,9)$.

Por otro lado, se evidenciaron inequidades geográficas en el acceso a los servicios de ecografía obstétrica y en la calidad del diagnóstico de los mismos, para las madres provenientes de las zonas rurales y de los municipios más apartados de la capital provincial (Cali). Aunque hallazgos similares se han reportado en países desarrollados $(11,12)$, la necesidad de lograr una adecuada regionalización de los servicios de atención prenatal es más urgente en los países en desarrollo como Colombia $(14,15)$.
El hallazgo de un diagnóstico prenatal completo y exacto en solamente el $24,0 \%$ y de un diagnóstico incompleto o inexacto en el $11,6 \%$ de los RN con ACM hospitalizados en las UCIN estudiadas es similar al reportado por Gómez y cols (8), quienes establecieron la presencia de diagnóstico prenatal en el $32 \%$ de los RN con anomalías congénitas diagnosticadas antes del alta, y consistente con los hallazgos del estudio RADIUS de 1993(3), que reportó un diagnóstico prenatal de ACM en el $34,8 \%$ de un grupo de pacientes sometidas a tamizaje con EcoPN versus $11 \%$ en el grupo control.

En Latinoamérica, Campaña y cols (7), encontraron diagnóstico prenatal en el $56 \%$ de los casos en 18 hospitales de 4 países. Aunque las metodologíasde estos estudios fueron diferentes, todos evaluaron el diagnóstico prenatal de ACM. Nuestro estudio no incluyó casos de RN con ACM fallecidos antes de ser ingresados a las UCIN, o con ACM que no fueron lo suficientemente graves para requerir hospitalización. Es de notar que los resultados presentados en este trabajo están dentro del rango de otros que han evaluado diagnósticos prenatales (6-9), lo cual valida los hallazgos de este estudio.

El Estado y los entes de control de los aseguradores privados en salud deben garantizar que todas las mujeres embarazadas tengan acceso a ecografías obstétricas de buena calidad, sin importar su condición socioeconómica o área de procedencia, llevando a las instituciones de salud ubicadas en municipios alejados y en zonas rurales especialistas capacitados y bien remunerados, con ecógrafos de adecuada tecnología, incluso disponiendo de ecógrafos portátiles donde se requieran, así, se podrá mejorar la cobertura y la calidad de la ecografía obstétrica en Colombia para cerrar las disparidades encontradas.

\section{CONCLUSIONES}

En las mujeres incluidas en el estudio se encontraron inequidades en la realización de ecografías obstétricas y en el diagnóstico de anomalías congénitas mayores para las madres afiliadas al régimen de salud subsidiado o sin afiliación, para las madres procedentes de municipios diferentes a la capital de la provincia y para las madres provenientes de áreas rurales, evidenciando que la accesibilidad al control prenatal y a la ecografía obstétrica es deficiente para las mujeres pobres y para aquellas que habitan en las áreas más apartadas de los centros urbanos, donde se concentran las tecnologías en salud.

\section{REFERENCIAS}

1. Castilla E, Orioli IM. ECLAMC: The Latin-American Collaborative Study of Congenital Malformation. Community Genet 2004;7:76-94. Gregory F. Genetic after 
of development. En: Nussbaum R, McInnes R, Willard $\mathrm{H}$. Thompson and Thompson Genetics in Medicine. 6th ed. Philadelphia: WB Saunders Co; 2001. pp 33557.

2. Whitworth M, Bricker L, Neilson JP, Dowswell T. UItrasound for fetal assessment in early pregnancy. Cochrane Database Syst Rev 2010;(4):CD007058.

3. Ewigman B, CraneJ, Frigoletto F, LeFevre M, Bain R, McNellis D. Effect of prenatal ultrasound screening on perinatal outcome. N Engl J Med 1993;329:821-7.

4. Saldarriaga W, Artuz A. Ayudas Diagnosticas en Obstetricia. En: Fundamentos en Ginecología y Obstetricia. Cali - Colombia: Universidad del Valle Programa Editorial; 2010. pp 264 - 77.

5. Deprest J, Flake A, Gratacos E, Ville Y, et al. The making of fetal surgery. Prenatal Diagnosis 2010;30(7):653-67.

6. Stoll C, Clementi M. Prenatal diagnosis of dysmorphic syndromes by routine fetal ultrasound examination across Europe. Ultrasound Obstet Gynecol 2006;21:543-51.

7. Campaña H, Ermini M, Aiello H, Krupitzki H, Castilla E, López-Camelo J. Prenatal sonographic detection of birth defects in 18 hospitals from South America. J Ultrasound Med 2010;29:203-12.

8. Gómez J, Fernández N, Páez P, Zarante I. Detección de anomalías congénitas en 12760 nacimientos de tres hospitales en la ciudad de Bogotá, Colombia 2004-2005, mediante ecografía prenatal. Rev Colombiana Obstet Ginecol 2007;58:194-201.

9. Saldarriaga-Gil W, Ruiz-Murcia FA, Fandiño-Losada A, Cruz-Perea M. E, Isaza de Lourido C. Evaluation of prenatal diagnosis of congenital defects by screening ultrasound, in Cali, Colombia. Colombia Médica 2014;45:32-8.

10. Peiris V, Singh TP, Tworetzky W, Chong EC, Gauvreau K, Brown DW. Association of socioeconomic position and medical insurance with fetal diagnosis of critical congenital heart disease. Circ Cardiovas Qual Outcomes 2009;2:354-60.

11. Maxwell S, Brameld K, Bower C, Dickinson JE, Goldblatt J, Hadlow N, et al. Socio-demographic disparities in the uptake of prenatal screening and diagnosis in Western Australia. Aust N Z J Obstet Gynaecol 2011;51:9-16.
12. Heaman MI, Green CG, Newburn-Cook CV, Elliott LJ, Helewa ME. Social inequalities in use of prenatal care in Manitoba. J Obstet Gynaecol Can 2007;29:806-16.

13. Smith LK, Budd JL, Field DJ, Draper ES. Socioeconomic inequalities in outcome of pregnancy and neonatal mortality associated with congenital anomalies: population based study. BMJ 2011;343:d4306.

14. Say $L$, Raine R. A systematic review of inequalities in the use of maternal health care in developing countries: examining the scale of the problem and the importance of context. Bull WHO 2007;85:812-19.

15. Houweling TA, Ronsmans C, Campbell OM, Kunst $\mathrm{AE}$. Huge poor-rich inequalities in maternity care: an international comparative study of maternity and child care in developing countries. Bull WHO 2007;85:74554.

16. Victoria CG, Matijasevich A, Silveira MF, Santos IS, Barros AJD, Barros FC. Socio-economic and ethnic group inequities in antenatal care quality in the public and private sector in Brazil. Health Policy Plan 2010;25:253-61.

17. Homedes N, Ugalde A. Why neoliberal health reforms have failed in Latin America. Health Policy 2005;71:83-96.

18. Reichenheim ME. Confidence intervals for the kappa statistic. Stata J 2004;4:421-8.

19. Sudhakaran N, Sothinathan U, Patel S. Best practice guidelines: Fetal surgery. Early Human Develop 2012; 88:15-9.

20. Simpson JL, Greene MF. Fetal SurgeryforMyelomeningocele? N Engl J Med 2011;364:1076-7.

21. Adzick S, Thom E, Spong C, Brock J, et al. A randomized trial of prenatal versus postnatal repair of myelomeningocele. N Engl J Med 2011;364:993-1004.

22. Holland A, Walker K, Badawi N. Gastroschisis: an update. Pediatr Surg Int 2010;26:871-8.

23. Norma técnica para la detección temprana de las alteraciones del embarazo. Ministerio de protección social. 2000. Bogotá DC.

24. Ministerio de Salud y Protección Social-Colciencias. Guías de práctica clínica para la prevención, detección temprana y tratamiento de las complicaciones del embarazo, parto o puerperio. Guía No. 11-15. Bogotá, Colombia; 2013. 